\title{
Prescrição de exercícios físicos por inteligência artificial: a educação física vai acabar?
}

\author{
Prescription of exercises by artificial intelligence: is this the end of physical education? \\ Prescripción de ejercicios físicos por inteligencia artificial: ¿acabará la educación física? \\ Braulio Nogueira de Oliveira ${ }^{\text {(1D) }}$, Alex Branco Fragab,c (i)
}

Palavras-chave:

Educação física e treinamento; Inteligência artificial; Exercício físico; Internet.

\begin{abstract}
RESUMO
Neste ensaio, tratamos de aplicativos fitness que, autonomamente, prescrevem exercício físico e algumas possíveis repercussões para a Educação Física. A prescrição por meio de inteligência artificial articula um conjunto de variáveis para a elaboração do treinamento, aproximando-se do que denominamos de "protocolização das práticas corporais". Considerando as características da tecnologia em questão, elencamos três pontos de reflexão aos profissionais de Educação Física: não restringir o sujeito a variáveis; não se subordinar a padrões preestabelecidos; e, por fim, reconhecer a potência do encontro. Logo, à máquina cabe articular um conjunto de variáveis; aos profissionais, desenvolver competências eminentemente "humanas".
\end{abstract}

\section{Physical education} and training; Artificial intelligence; Exercise; Internet.

Palabras clave: Educación y entrenamiento físico; Inteligencia artificial; Ejercicio físico; Internet.

\begin{abstract}
This essay deals with fitness applications which, autonomously, prescribe physical exercise and some possible repercussions for Physical Education, approaching what we call "protocolization of body practices". Considering the characteristics of the technology in question, we have listed three points of reflexion for Physical Education professionals: to not restrict the subject to variables, to not subordinate to pre-established standards, and, finally, to recognize the importance of social interaction. Therefore, the machine is responsible for articulating a set of variables whereas the professionals are responsible for developing eminently 'human' skills.
\end{abstract}

\section{Keywords:}

\section{RESUMEN}

En este ensayo tratamos acerca de aplicaciones de fitness que, de forma autónoma, prescriben el ejercicio físico y algunas posibles repercusiones para la Educación Física. La prescripción mediante el uso de la inteligencia artificial articula un conjunto de variables para la elaboración del entrenamiento, aproximándose así a lo que denominamos "protocolización de las prácticas corporales". Considerando las características de la tecnología en cuestión, hemos enumerado tres puntos de reflexión para los profesionales de la Educación Física: no restringir el tema a variables; no subordinarse a estándares preestablecidos; $y$, finalmente, reconocer el potencial del encuentro. Por tanto, la máquina se encarga de articular un conjunto de variables; a los profesionales, desarrollar habilidades eminentemente "humanas".

\footnotetext{
aColégio Militar de Fortaleza, Seção de Educação Física. Fortaleza, CE, Brasil.

'Universidade Federal do Rio Grande do Sul, Programa de Pós-graduação em Ciências do Movimento Humano. Porto Alegre, RS, Brasil. 'University of Toronto, Faculty of Kinesiology \& Physical Education - FKPE, Toronto, Ontario, Canada.
}

\footnotetext{
*Autor correspondente:

Braulio Nogueira de Oliveira

E-mail brauliono08@hotmail.com
} 


\section{INTRODUÇÃO}

"O personal trainer mais popular do país não é humano." Esse é o título de um texto publicado na revista Veja ${ }^{1}$, que remete a pensar sobre o emprego das tecnologias digitais, especialmente as tecnologias disruptivas, na prática de exercícios físicos, de modo geral, quanto na Educação Física, de modo particular. Conforme o próprio nome sugere, disruptividade significa a ruptura com "produtos" já estabelecidos (Utterback e Acee, 2005). Há diversos exemplos de tecnologias que modificaram o modo como as pessoas se relacionam com serviços, como o de transporte (com a emergência da Uber); hospedagem (com a emergência da Airbnb); bancário (com a emergência da Nubank); locação de filmes (com a emergência da Netflix). A reportagem da revista Veja trata de um aplicativo fitness que, autonomamente, prescreve exercícios físicos. Logo, modifica o modo de as pessoas se relacionarem com os exercícios físicos e, consequentemente, provoca adaptações para a Educação Física.

Ao detalhar características do personal trainer, o subtítulo desse mesmo texto publicado na revista Veja diz: "Sua capacidade de aprender com o feedback dos alunos e criar programas de treinamento físico personalizado é um dos seus segredos". Portanto, trata-se de algo que "não é humano" e, ao mesmo tempo, tem "capacidade de aprender", ou seja, o personal trainer em questão é um software baseado em inteligência artificial: um personal trainer digital. Isso implica dizer que o aplicativo fitness é aprimorado, na medida em que mais dados são disponibilizados. Portanto, difere dos aplicativos fitness já conhecidos, nos quais os treinos já se encontram prontos e são apenas replicados para diferentes usuários.

Basicamente, a inteligência artificial permite o processamento extremamente rápido de grande quantidade de dados, de distintas fontes, e os resultados podem ser transmitidos às pessoas (Joshi, 2020), seja de dados individuais, ou mesmo de vários usuários simultaneamente - análise de big data. Permite, portanto, que o treinamento seja, de fato, personalizado, já que a prescrição é ajustada de acordo com os dados do usuário.

Não se pode dizer que se trata de algo propriamente novo, já que Delpizzo (1997) estudou a prescrição de atividades físicas através de inteligência artificial ainda na década de 1990, em dissertação defendida no Programa de Pós-graduação em Engenharia de Produção da Universidade Federal de Santa Catarina (UFSC) ${ }^{2}$.

1 Por Abril Branded Content, publicado em 7 de junho de 2017, na revista Veja, Editora Abril. Disponível em: https://veja.abril.com.br/especiais/o-personal-trainermais-popular-do-pais-nao-e-humano/. Acesso em: 2 fev. 2020.

2 Ironicamente, a autora, Vanessa Lins Francalacci Delpizzo,
Tampouco que abrange somente a área da Educação Física, já que, dentre diversas áreas, o futuro da Medicina e da Educação Médica também se encontra em debate como decorrência da capilarização da inteligência artificial (Lobo, 2018).

Uma experiência particular vivenciada por um dos autores deste texto permite dimensionar a perfusão dos aplicativos fitness em distintas áreas. Em dezembro de 2019, uma amiga com disfunção na tireoide, com dificuldade de emagrecimento, foi atendida por um profissional endocrinologista. Adepta de uma vida fitness, realizava suas práticas de treinamento resistido e treinamento aeróbico em tradicional clube de uma das capitais nordestina. $\mathrm{O}$ endocrinologista questionou se ela gostava de usar aplicativos. Após resposta positiva, escreveu na receita, dentre outras coisas, o seguinte encaminhamento: \# experimentar o aplicativo MyFitnessPal. Trata-se de um aplicativo de self-tracking que se vincula a outros, por meio do qual é possível registrar aspectos da vida diária, como o consumo e o gasto calórico. Essa vivência demarca uma mudança crucial no âmbito da cultura fitness: no primeiro momento, o endocrinologista não recomendou caminhada ou seguir a orientação de um profissional de Educação Física, mas sim o uso do aplicativo.

O texto publicado na revista Veja trata, especificamente, de um aplicativo fitness vinculado à empresa alemã Freeletics, amplamente difundido no mundo. O personal trainer digital (chamado também de Coach, ou Coach digital, nas publicações da empresa), conta com cerca de 40 milhões de usuários (Freeletics, 2020). Portanto, reúne uma quantidade de usuários superior à que qualquer personal trainer "de carne e osso" poderia reunir. O software personaliza um treinamento que considera os dados de todos esses usuários em conjunto (big data), assim como os dados individuais. Envolve desde características pessoais (idade, peso, objetivo) à Percepção Subjetiva do Esforço após os treinos (por meio do feedback pessoal, em uma escala de resposta psicométrica). Para além da prescrição personalizada de exercícios físicos, intencionalmente ou não, o personal trainer digital, os outros atores humanos e não humanos, integram uma rede sociotécnica, que produz imperativos para uma vida fitness, sugerindo que os usuários sejam: inspiração, self-trackers, mais fortes que sua melhor desculpa e empreendedores de si (Oliveira, 2021).

Evidentemente, a emergência desse tipo de tecnologia desloca uma condição aparentemente estável e confortável aos profissionais de Educação Física e, nesse sentido, nos permite, neste ensaio, contribuir com o debate que transita pelo trinômio exercícios físicos, tecnologia e Educação Física. Nesse sentido, o presente ensaio tem por objetivo discutir repercussões da

foi conselheira efetiva do CREF3/SC na gestão de 20032017, entidade diretamente afetada com a emergência dessa tecnologia. Disponível em: https://www.crefsc.org. br/diretoria-2003-2005/. Acesso em: 29 set. 2020. 
emergência de um personal trainer digital para a área da Educação Física, evidenciando alguns pontos de reflexão.

\section{REPERCUSSÕES DO PERSONAL TRAINER DIGITAL: A EDUCAÇÃO FÍSICA VAI ACABAR?}

A repercussão de uma tecnologia que, autonomamente, prescreve exercícios físicos, demanda considerar uma série de mudanças em distintas áreas da produção de bens e serviços. O debate acerca da indústria 4.0, ou mesmo do que vem sendo chamado de indústria 5.0 , perpassa por algumas transições interessantes em relação a uma evolução das tecnologias. Grosso modo, essas expressões reportam o que pode vir a ser a quarta ou a quinta revolução industrial, considerando já ter havido a primeira, em que máquinas substituíram a força animal; a segunda, com a energia elétrica e linha de produção; e a terceira, com a modernização do trabalho.

Uma característica comum entre todas elas é a sofisticação das máquinas, capazes de desenvolver funções cada vez mais complexas, justamente o que diferencia a terceira da quarta revolução ${ }^{3}$. Já a quinta revolução industrial, ou a Sociedade 5.0, refere-se a uma aproximação entre homem e máquina, conforme o fragmento a seguir que consta no site Informa Markets: "A Indústria 5.0 funde a criatividade e a habilidade humana com a velocidade, a produtividade e a consistência dos robôs. Deste modo, os sistemas inteligentes, ao invés de inimigos, passam a contribuir [...]"4.

Essa transição nos dá subsídios para pensar os mesmos processos para a Educação Física. Evidentemente, a citação anterior demonstra uma visão bastante otimista quanto à projeção desse cenário, desconsiderando que a indústria 4.0, ou mesmo a suposta emergência de uma indústria 5.0, pode aprofundar alguns problemas, como a precarização das relações de trabalho.

Por outro lado, compreendemos que parte do que é realizado pelo profissional de Educação Física, assim como em diversas outras categorias profissionais, provavelmente será (e, em certa medida, tem sido) desempenhado pela máquina. Nesse processo, aquilo que é considerado humano e não humano, muitas vezes, aparenta ter característica híbrida. Ora, assim como alguns profissionais de Educação Física, o personal trainer digital é normalmente chamado de coach.

3 Por Fia. Indústria 4.0: o que é, consequências, impactos positivos e negativos [Guia Completo]. Em 6 de julho de 2018. Disponível em: https://fia.com.br/blog/ industria-4-0/. Acesso em: 27 set. 2020.

4 Por Informa Markets. Indústria 5.0: A reconciliação entre o homem e a máquina. Em 12 de fevereiro de 2020. Disponível em: https://saudebusiness.com/voceinforma/industria-5-0-a-reconciliacao-entre-o-homem-ea-maquina/. Acesso em: 27 set. 2020.
Originalmente, coach é um termo de língua inglesa que, dentre algumas traduções possíveis, significa treinador/a. Basicamente, consiste em uma pessoa que utiliza "técnicas de coaching" para preparar/aperfeiçoar alguém em alguma área específica. A princípio é mais utilizado para representar treinadores esportivos, o que remete a pensar que há uma competição em voga.

Essa racionalidade competitiva é também cooptada para ressignificar o que vem a ser o coach no contexto da cultura do empreendedorismo, não mais restrito a treinadores esportivos. Nessas outras áreas, para exercer a função de coach, ter uma formação superior não é requisito, motivo pelo qual, aliado ao fato de abranger diversas áreas (como, por exemplo, coach de relacionamentos), é um profissional muito satirizado.

Há diversos memes, piadas e afins relacionadas a essa prática, tais como algumas páginas na internet intituladas "Coach nem é gente". Sem entrar no mérito do profissionalismo ou não dos coaches, pode-se dizer que, agora, essa frase, em alguns casos, deixa de ser meramente cômica e passa a ser um fato: há softwares, como o personal trainer digital, que são coaches, logo, de fato, alguns nem são gente.

Ironicamente, embora sem relação com o primeiro meme e sim com as condições de trabalho, outra frase recorrente nas mídias sociais, é: "Professor também é gente". Isso permite reformular a primeira definição proposta nesse mesmo parágrafo: Coach consiste basicamente em uma pessoa, ou software, que utiliza "técnicas de coaching" para preparar alguém, para algo. Obviamente essas "técnicas de coaching" também são diversas; no caso do personal trainer digital, são especialmente relacionadas à vida fitness. Há outros aplicativos que envolvem mais aspectos da vida, como é o caso da propaganda em vídeo divulgada no Instagram, da qual apresentamos a transcrição do conteúdo do áudio.

Oi, nós somos o [...]. E nós usamos a ciência para melhorar o seu bem-estar.

Você sabia que naturalmente nós estamos inclinados a repetir ações diariamente? Seja a hora de acordar pela manhã ou a xícara na qual você bebe seu café... Todos nós estamos fadados a repetir o mesmo comportamento. Também conhecido como... hábitos

Hábitos? Bem, eles podem ser bons. Mas também podem ser ruins. Então, se nós não podemos evitar os hábitos, como mantê-los bem? É aí que entra o [...]. Nós somos experts nisso. Nós te ajudamos a manter os hábitos saudáveis firmes. Usando técnicas de ciência comportamental desenvolvidas na Universidade de Duke, nós organizamos os exercícios para você que te ajudam a construir hábitos saudáveis. $E$ nós não apenas te ajudamos a construir coisas pequenas como beber água, nós desenvolvemos planos para que você alcance metas de vida mais altas - usando os hábitos que nós vamos te ensinando pelo caminho. 
Nós somos o [...]. E vamos usar a ciência para melhorar o seu bem-estar.

Vamos lá, dê uma chance a nós. ${ }^{5}$

Essa publicação chama a atenção justamente por sua pretensa perfusão na rotina diária, no sentido de direcionamento de comportamentos, bem como sua vinculação com a ciência. Em tese, esse coach orienta desde os hábitos mais simples, como beber água, até outros, mais complexos, como a prática de exercícios físicos. A princípio, vale reiterar que a emergência dessas tecnologias abrange as diferentes áreas. Têm reconfigurado os mais distintos setores e, assim, evidencia ainda mais a necessidade de reflexão. Nesse sentido, enquanto professores e profissionais de Educação Física, vale pensar em nossas características que se colocam como limites, bem como nossas potencialidades, por entender que alguns delas talvez sejam transitórias, ao passo em que outras são permanentes. Nesse cenário, surgem diversos questionamentos, dentre os quais, na emergência de um personal trainer digital, um deles ganha relevo especial: a Educação Física vai acabar?

Essa problematização remete a pensar um denso e complexo debate, que historicamente evidencia tensões na área, em torno da seguinte questão: A atuação em Educação Física manifesta-se apenas de uma forma? Nesse momento, investimos nessa discussão do ponto de vista estritamente de atuação profissional e não curricular (se o curso deveria ser licenciatura única ou bacharelado e licenciatura $)^{6}$. Particularmente, como fruto de análises empreendidas no contexto brasileiro, temos pelo menos três subáreas: biodinâmica, pedagógica e sociocultural (Manoel e Carvalho, 2011). Em análise mais ampla, poderíamos alocá-las em duas: ciências da natureza, da qual deriva a subárea biodinâmica; e ciências sociais, da qual derivam as subáreas pedagógica e sociocultural.

Ainda que tanto as ciências da natureza quanto as ciências sociais tenham a contribuir com a área, produzindo uma Educação Física mais potente, ou mesmo "uma só", há propostas distintas, marcadas pelas características metodológicas de cada uma e que reverbera na atuação pedagógica profissional. A evidente relação entre epistemologia e prática pedagógica mostra-se mais fortemente nas tendências pedagógicas da Educação Física, em que temos diversas propostas fundamentadas em determinada perspectiva epistemológica e, consequentemente, resulta em

5 Texto de divulgação do aplicativo Fabulous, disponível em vídeo na conta do Instagram da empresa.

6 Vale ressaltar que essa possível divisão do curso em licenciatura e bacharelado é objeto de discussão muito forte no campo da formação em Educação Física, embora não estejamos tratando dela neste texto. modos singulares, alinhados ao autor de base, para operacionalizar a prática.

Logo, entende-se que a epistemologia é "[...] indissociável da metodologia uma vez que o estudo da ciência é também o estudo dos seus métodos." (Mendes et al., 2009, p. 92). Nesse sentido, compreendemos que o personal trainer digital, por exemplo, possui características mais alinhadas a determinadas perspectivas epistemológicas, em detrimento de outras.

Apesar de existirem exceções, o campo das ciências da natureza pressupõe maior neutralidade; distanciamento entre pesquisador e objeto; lidar mais com números e análises estatísticas. O cerne do debate permeia, na maioria das vezes, a dimensão biológica, mensurável, reproduzível. Assim sendo, a análise de certas variáveis nos permite a tomada de decisão, na esteira do campo de estudos e práticas denominado Práticas Baseadas em Evidência. Já o campo das ciências sociais pressupõe maior posicionalidade; uma aproximação entre pesquisador e objeto; ao lidar mais com informações qualitativas. O cerne do debate permeia, na maioria das vezes, a dimensão socio-políticocultural, não mensurável e singular.

De certo modo, por vezes, o embate entre as duas ciências embarreirou o avanço da área, enquanto em algumas outras ocorreram diálogos interessantes. Assim sendo, vale retomar cerca de 20 anos da publicação de um texto de Yara Maria de Carvalho (2001), de título sugestivo: Atividade Física e Saúde: Onde Está e quem É o 'Sujeito' da Relação?. Assim, apresentamos o argumento da autora:

O "lugar" destinado ao sujeito, ou o entendimento que prevalece a respeito do sujeito está caracterizado por uma "figura" que muitas vezes não pensa, não sente, não experimenta emoções, desejos, não carrega consigo sua própria história de vida. Frequentemente ele aparece escondido em um grupo de sedentários ou praticantes de atividade física; em um grupo definido pela faixa etária (adulto, idoso); em um grupo definido pela natureza da atividade física que desenvolve; atleta, não-atleta; indivíduos sadios e doentes, entre outras conotações. (Carvalho, 2001, p. 10).

A autora faz uma crítica à forma como os "sujeitos" são tratados nos estudos, considerados como "amostra". A noção de "amostra" é colocada como uma porção de algo - no caso dos estudos, de uma dada "população"; logo, a pessoa é reduzida ao conjunto de dados produzidos a partir dela, normalmente a ser tratado estatisticamente. A preocupação da autora não se dá exatamente pela semântica do termo, mas pela reprodução dessa noção de "amostra" no campo de atuação (e não somente de estudos), quando as pessoas são reduzidas a seus dados. Alerta que certas variáveis dão corpo ao sujeito: idade, nível de aptidão física, condições de saúde, entre algumas outras poucas. Desse modo, aponta que reduzir o sujeito 
a essas informações o aproxima mais de um boneco do que propriamente um ser humano (Carvalho, 2001). Na lógica dessa crítica, o sujeito ${ }^{7}$ é posto em segundo plano, escondido atrás de suas próprias variáveis; precisamente, é reduzido a suas variáveis.

Recentemente, um dos autores deste texto identificou a propaganda de um curso que prometia a prescrição de exercícios físicos com base exclusivamente em exames laboratoriais, "este, sim, baseado em evidências" - dizia o anúncio. Ora, se o sujeito está em segundo plano, em detrimento de suas variáveis, ou se ele se resume a suas variáveis, há alguma dúvida de que a máquina prescreverá o treino mais adequado? Com efeito, se a prescrição restringe-se a manipular um conjunto de variáveis e aplicar outro conjunto de fórmulas para obter a receita ideal, o software, definitivamente, faz melhor! Isso não somente na área da Educação Física.

Em artigo publicado na revista Folha de S. Paulo, em janeiro de 2020, por Fergus Walsh, foi divulgado um artigo publicado na revista Nature no qual se afirma que a inteligência artificial foi mais eficiente do que médicos radiologistas no diagnóstico de câncer de mama, a partir do exame de mamografiå.

A inteligência artificial poderá (em certa medida, pode), sem muitas dificuldades, inclusive, identificar as evidências científicas (de uma ciência específica, vale a ressalva) e indicar o melhor caminho. Revisões padronizadas, como as recomendações dos Principais Itens para Relatar Revisões sistemáticas e Metaanálises (Prisma), já classificam aqueles estudos considerados mais relevantes, a depender do tratamento estatístico empregado, que padronizam o modo de escrita e apresentação dos resultados, como, até mesmo, facilitadores do trabalho das máquinas.

Nesse sentido, entendemos que, no campo das Práticas Baseadas em Evidências, considerando as empregadas nas ciências da natureza, enquanto mais relevantes, em relação àquelas das ciências sociais, como sugere a escala de qualidade da evidência, pode facilmente embasar atividades realizadas pela máquina. O método Grading of Recomendations Assessment, Developing and Evaluation (Grade), por exemplo, aloca os estudos experimentais e aqueles com

7 Por questões epistemológicas, não procuramos, na noção de "sujeito", uma essência humana (fenomenológica, humanista), ao passo que nos distanciamos de uma compreensão que parte de uma dada "natureza humana", em decorrência de uma constituição pela linguagem (uma "posição-sujeito").

8 Por Fergus Walsh, em janeiro de 2020, intitulado Câncer de mama: Inteligência artificial bate médicos em diagnósticos, publicado na revista Folha de $S$. Paulo. Disponível em: https://www1.folha.uol.com.br/ equilibrioesaude/2020/01/cancer-de-mama-inteligenciaartificial-bate-medicos-em-diagnosticos.shtml. Acesso em: 12 abr. 2020. desfechos provenientes de ensaios clínicos randomizados como mais relevantes (Galvão e Pereira, 2015). Nessa lógica, estudos que não permitem uma revisão do tipo metanálise, como os qualitativos, são menos relevantes.

Propomos a expressão "protocolização das práticas corporais", para definir o estabelecimento de condutas prévias, em função de certa quantidade de variáveis, geralmente limitadas. Vale ressaltar que seria prática facilmente reproduzida pelo software. E mais... caso a escuta se limite a outras variáveis, como um feedback em escala, como a de Borg, também estaríamos superados. Não queremos, com isso, deslegitimar a adoção de protocolos, mas problematizar o seu uso indiscriminado. Um protocolo serve como orientação, e não como restrição de outros possíveis.

Historicamente, teóricos do campo das subáreas sociocultural e pedagógica, na Educação Física, problematizam essas relações, especialmente no campo da saúde. Particularmente nesse campo, o que chamamos de protocolização das práticas corporais tem sido denominado, no Brasil, de medicalização das práticas corporais (ou algo similar, como medicalização da atividade física, da educação física, etc.).

No âmbito de estudos sobre atividade físicas/ exercícios físicos para grupos especiais, por exemplo, há tabelas que compreendem o sujeito pela patologia que o acomete, e indica que exercícios devem ser prescritos para cada grupo, de acordo com a patologia (Pedersen e Saltin, 2015). Embora relevante no sentido de compreender os parâmetros para a prescrição de exercícios físicos em dadas condições fisiológicas, esse tipo de abordagem torna-se problemática pelo fato de que o sujeito não se restringe à patologia diagnosticada; e também porque dificilmente o usuário é acometido por somente uma daquelas condições patológicas.

Assim sendo, um indivíduo que se encontra ao mesmo tempo obeso, hipertenso e sofre por ansiedade, deverá seguir a dosagem de exercício físico para qual dessas condições? Se essas variáveis forem suficientes, o aplicativo exercerá bem a prescrição de exercícios físicos.

Há décadas, os teóricos do campo das ciências da natureza procuram consolidar práticas baseadas em evidências, que são, conforme já mencionado, hierarquizadas a depender do tratamento estatístico empregado. Finalmente, temos um coach disposto a prescrever exercícios físicos com base exclusivamente nessas variáveis: o aplicativo fitness, munido de inteligência artificial. $O$ diferencial é simples: em vez de uma receita única para todos, o personal trainer digital possui um conjunto de receitas, customizáveis de acordo com um conjunto de variáveis coletadas pelos aplicativos - uma protocolização das práticas corporais.

A rigor, compreendemos que a tecnologia pode assumir um caráter complementar, contemplando limitações do profissional, como a identificação automática do consumo calórico diário, realizadas por smartwatch, que pode vir a orientar os profissionais de 
Educação Física. No contexto da prescrição automática de exercícios físicos, embora possibilite acesso a algum tipo de orientação para pessoas que não possuem condições, sejam informacionais ou financeiras, de contratar um professor de Educação Física, a tecnologia possui limites, conforme destacamos.

Assim considerando, elencamos três pontos de reflexão aos profissionais de Educação Física, a serem discutidas na sequência: Não restringir o sujeito a variáveis; reconhecer a potência do encontro; e não se subordinar a padrões preestabelecidos. Aparentemente, são sugestões com tom prescritivo e cunho moralista, contudo, nossa ideia é que soem como propositivas e, especialmente, provocativas, e não exatamente um "faça!".

Assim sendo, o primeiro ponto de reflexão é "não restringir o sujeito a variáveis". Recordo-me de vários amigos que relataram ir a consultas médicas sem sequer serem olhados - apenas algumas de suas variáveis eram vistas. Lembro-me ainda de um que disse ter sido tratado dessa mesma forma, em um grupo de corrida. O profissional apenas apresentou um conjunto de questões já prontas em uma planilha, prescrevendo o treinamento na sequência, sem sequer olhá-lo antes do treino, e considerar leve a dor no joelho que disse ter relatado estar sentindo antes da prática.

A cultura self-tracking, de modo geral, remete a pensar que os dados são representativos de quem o usuário é, inclusive, a expressão "you are your date" (ou "você é seus dados", em tradução livre), integra o título de uma das obras de autores como Deborah Lupton (2016). Na perspectiva aqui proposta de não restringir o sujeito a variáveis, torna-se oportuno discordar dessa afirmação. Se formos somente nossos dados, nossas variáveis, a inteligência artificial poderia, certamente, prescrever exercícios com maior precisão.

Por outro lado, é preciso também conhecer e não desprezar as variáveis. Um meio de efetivar a não restrição do sujeito é a chamada, no campo da saúde coletiva escuta qualificada, ou mesmo o que, no campo da educação, denominamos de aprendizagem significativa, ou seja, tornar relevante aquilo que faz sentido ao sujeito, sua subjetividade, seus aspectos particulares, inclusive os não mensuráveis.

Uma ressalva importante é que os algoritmos baseados em inteligência artificial também conseguem "ler" argumentos textuais, ou seja, não numéricos. No debate acerca da disruptividade da tecnologia, isso se torna ainda mais pertinente, na medida em que já existem, por exemplo, Instituições de Ensino Superior (IES) vinculadas à rede educacional Laureate, que fazem uso de inteligência artificial para correção de textos e atribuição de notas em curso de Educação a Distância $(E a D)^{9}$. O mais

9 Por Thiago Domenici. Laureate usa robôs no lugar de professores sem que alunos saibam. Publicado em 30 abr. 2020. Disponível em: https://apublica.org/2020/04/ laureate-usa-robos-no-lugar-de-professores-sem-que- interessante disso é que alguns alunos sequer perceberam que estavam sendo avaliados por um software.

Um segundo ponto de reflexão é "reconhecer a potência do encontro". Ainda que a máquina condicione um encontro de si consigo mesmo, uma espécie de autoconhecimento através dos números, o contato pessoal com o profissional da área, ou mesmo com outros praticantes, possibilita um aprofundamento até mesmo desse relacionamento consigo mesmo.

O produto do encontro entre esses diferentes atores não se limita à soma de suas experiências, mas envolve algo novo, constituído pela multiplicação de potencialidades engendradas por experiências singulares, que podem vir a superar certas limitações que são de um, mas não de outro.

A propósito, a inteligência artificial possui limitações. Considerando que agrupa dados disponíveis, reproduz alguns aspectos problemáticos da cultura, a exemplo da robô Tay, um chatbot criado pela Microsoft com o objetivo de interagir com jovens no Twitter e "aprender" com essa interação. Tay precisou ser inativada em poucas horas, pois, pela influência da cultura, as informações apreendidas a fez se tornar racista, sexista e xenófoba. Ora, à maioria dos chatbot, são atribuídos o gênero feminino por influência da cultura. A inteligência artificial alimenta-se exclusivamente de informações disponibilizadas, sem uma criticidade mais profunda.

Um terceiro ponto de reflexão é "não se subordinar a padrões preestabelecidos", a protocolizar as práticas, o que não pode, paradoxalmente, se constituir como um padrão. Referimo-nos a situações como a sensibilidade eminentemente humana de identificar que, em determinado dia problemático para o usuário, é possível que uma caminhada na praça seja melhor do que o treinamento resistido na academia de musculação. Os protocolos servem para orientar, mas não para acorrentar.

O trabalho pautado no "piloto automático", sem planejamento, replicando prescrições, é facilmente superado pela máquina. Nossos pontos de reflexão, portanto, são no sentido de a máquina exercer as funções que demandam que estejamos no piloto automático, de caráter complementar, para que possamos cuidar da dimensão "humana", daquilo que não é possível mensurar. Trata-se, portanto, de um convite a explorar a dimensão da criatividade, expressão em que a inteligência artificial é limitada.

\section{CONSIDERAÇÕES FINAIS}

Neste ensaio, discutimos as repercussões do emprego de tecnologias capazes de prescrever exercícios físicos por meio de inteligência artificial, para a Educação Física. O debate permeia a noção de ciência, considerando que análises semelhantes àquelas realizadas pela ciência da natureza, particularmente as complexas análises

alunos-saibam/\#.XqrvvKaQleM.twitter. Acesso em: 19 out. 2020. 
estatísticas, inclusive baseadas em evidências, poderão ser feitas pela máquina. Propomos o distanciamento de uma possível protocolização das práticas corporais, ou seja, o estabelecimento de condutas prévias, em função de certa quantidade de variáveis, geralmente limitadas. Considerando o contexto da Indústria 5.0, em que as máquinas podem vir a potencializar competências humanas, sem qualquer pretensão determinista ou moralista, evidenciamos três pontos de reflexão para os profissionais da área: não restringir o sujeito a variáveis; reconhecer a potência do encontro; e não se subordinar a padrões preestabelecidos.

Este debate deverá transitar por diversos campos de atuação da Educação Física, nos próximos anos. O modos operandi eminentemente técnico, baseado exclusivamente em evidências, articulado com diversas variáveis de milhões de usuários, surpreendentemente, pode ser executado pelas máquinas, pelo personal trainer digital. Aquelas características que podem ser consideradas propriamente humanas, como o diálogo, a escuta, criatividade, potência do encontro, o afeto, precisam ser reiteradas pelos profissionais. Dada a complexidade da atuação profissional, compreendemos que apenas parte das funções exercidas pelo profissional de Educação Física pode ser exercida pela máquina. Logo, entendemos que, talvez, apenas um "tipo" de profissional vá acabar. Apenas um "tipo" de Educação Física.

\section{FINANCIAMENTO}

O presente trabalho contou com uma bolsa de doutorado Capes.

\section{CONFLITOS DE INTERESSE} interesse.

Os autores declaram não haver conflitos de

\section{REFERÊNCIAS}

Carvalho YM. Atividade física e saúde: onde está e quem é o "sujeito" da relação? Rev Bras Ciênc Esporte. 2001;22(2):9-21.
Delpizzo VLF. Prescrição de atividades físicas através do uso da inteligência artificial [dissertação]. Florianópolis: Universidade Federal de Santa Catarina; 1997.

Freeletics [Internet]. Kit de Imprensa Munique. Munique, Alemanha: Freeletics GmbH; 2020 [citado em 2021 Fev. 15]. Disponível em: https://assets.contentstack.io/v3/assets/ blt45c082eaf9747747/blt0d51ff985bc6fa9e/5ef354a13a4 Od6578bbe9aaf/Projeto_Freeletics_PressKit_digital_PT.pdf

Galvão TF, Pereira MG. Avaliação da qualidade da evidência de revisões sistemáticas. Epidemiol Serv Saude. 2015;24(1):775-8. http://dx.doi.org/10.5123/S167949742015000100019.

Manoel EJ, Carvalho YM. Pós-graduação na educação física brasileira: A atração (fatal) para a biodinâmica. Educ Pesqui. 2011;37(2):389-406. http://dx.doi.org/10.1590/ S1517-97022011000200012.

Joshi AV. Machine Learning and Artificial Intelligence. Cham, Switzerland: Springer; 2020. http://dx.doi. org/10.1007/978-3-030-26622-6.

Lobo LC. Inteligência artificial, o Futuro da Medicina e a Educação Médica. Rev Bras Educ Med. 2018;42(3):3-8. http://dx.doi. org/10.1590/1981-52712015v42n3rb20180115editorial1.

Lupton D. You are your data: Self-tracking practices and concepts of data. In: Selke S, organization. Lifelogging: digital selftracking and lifelogging - between disruptive technology and cultural transformation. Wiesbaden: Springer VS; 2016. p. 61-79. http://dx.doi.org/10.1007/978-3-658-13137-1_4.

Mendes PC, Dias G, Morais F. A(s) Ciências do Desporto e o corpo: entre as Ciências Naturais e as Ciências Sociais. Exedra Rev Científica 2009;(1):91-100.

Oliveira BN. Personal trainer de bolso: uma tecnologia disruptiva na produção de imperativos para uma vida fitness [tese] Porto Alegre: Universidade Federal do Rio Grande do Sul; 2021.

Pedersen BK, Saltin B. Exercise as medicine - evidence for prescribing exercise as therapy in 26 different chronic diseases. Scand J Med Sci Sports. 2015;25(Suppl. 3):1-72. http://dx.doi.org/10.1111/sms.12581. PMid:26606383.

Utterback JM, Acee HJ. Disruptive technologies: an expanded view. Int J Innov Manage. 2005;1(1):1-17. 